\title{
Comparing Methods to Forecasting Var: Fhs Versus Evt Approaches
}

\author{
Panagiotis Delis ${ }^{1}$, John Hlias Plikas ${ }^{2}$ \\ ${ }^{1}$ University of Peloponnese, Msc in Economical analysis, Tripolis 22100, Greece
}

\begin{abstract}
Financial institutions have for many years sought measures, which identify and approach market risks in portfolios of financial instruments. Forecasting VaR has attracted a great deal of attention in the financial econometrics literature, the so-called filtered historical simulation (FHS) and Extreme Value Theory approaches. The paper provides a methodological contribution to the one-step-ahead VaR forecasting through some FHS and EVT approaches, which combine some conditional models for variance. Accounting asymmetrically distributed portfolio returns within the conditional variance process, accurate 99 per cent VaR forecasts are calculated for one-day-ahead horizon under Basel accords. After calculating VaR, we evaluate these methods for presenting the most appropriate methodology.
\end{abstract}

Keywords: Value-at-Risk, market risk, filtered historical simulation, EVT, efficient forecasting model

\section{Introduction}

Value-at-Risk ( $\mathrm{VaR})$ is the most popular tool in risk management and quantifies the maximum loss for a portfolio of assets under normal market conditions over a given period of time and at a certain confidence level. Forecasting VaR has attracted a great deal of attention in the financial econometrics literature, due to its large relevance for financial and insurance institutions. It is also important that the VaR measurement has been adopted by bank regulation. Under Basel accords the VaR methodology can be used by financial instruments to calculate capital charges in accordance with their financial risk. These institutions can determine their daily capital charge by following the three presequisites. A) The $99 \%$ confidence level must be used to hold the appropriate capital charge in order to ensure a safe and efficient market. B) The minimum holding period must be set to 10 trading days, so that investors are able to liquidate their positions. C) Financial institutions could calculate VaR by implementing internal models. The Basel Committee on Banking Supervision (2010) revised the proposed guidelines creating the Basel III. Within the literature the ability of a variety of increasingly complex models to estimate and forecast VaR has been tested. These models can account variously for certain features of financial asset/portfolio returns such as heteroskedasticity, asymmetry or leverage effects.These are a lot of reasons that many alternative specifications have been proposed including the stochastic volatility model (Taylor, 1982), the generalized autoregressive conditional heteroscedasticity (GARCH) model, the exponential generalized autoregressive conditional heteroscedasticity EGARCH model and the GJR-GARCH model, among many others. In order to derive the probability distributions of future returns implied by GARCH-type models, it is necessary to specify the distribution of the innovations.

There are different methods to estimate VaR, which can be classified into two main categories: parametric and simulation methods. These methods approach the conditional volatility and make assumption for distribution of asset/portfolio returns. Recently, a new methodology has been developed in the literature to estimate VaR. This methodology successfully combines bootstrapping techniques with the use of parametric models and is known as Filtered Historical Simulation (FHS). Under approach the bootstrap process is applied to the residuals of a time series model used as a filter to extract autocorrelation and heteroscedasticity from the historical time series of returns. FHS approach makes no assumption about the distribution of the returns under analysis, due to the fact that this method is based only on the assumption of IDD standardized residuals from an accurate volatility model, the use of the bootstrap algorithm allows a computationally feasible method to approach the unknown return empirical distribution.

However, the problem of the normality assumption of the return series can be addressed by applying extreme distribution based methods such as Extreme Value Theory (EVT). EVT provides a comprehensive theoretical base on which statistical models describing extreme scenarios can be formed. The Peak Over threshold method, which will be implemented in this paper, models a distribution of excess over a given threshold. EVT shows that this limiting distribution of exceedance is a Generalized Pareto Distribution (GPD) (Coles,2001; Handle and Hafner,2008; Gilli and Kellezi,2006). McNeil et al. (2000) first combined the GPD and the GARCH model to estimate conditional VaR. Marimoutou et al. (2009) applied the conditional GPD to the crude oil. Kourouma, Dupre, Sanfilippo and Taramasco et al. (2012) used conditional GPD in combination with GJR-GARCH model to forecast some stock indexes and commodity indexes. 
The purpose of this research is to investigate the predictive ability of FHS and EVT approaches in combination with the most accurate GARCH model, which captures the special features of return time series. Additionally, the evaluation framework is applied in order to compare and find the most efficient methodology to estimate and forecast $99 \%$ one-day $\mathrm{VaR}$ of four exchange rates (AUD/USD, EUR/JPY, EUR/USD and USD/CAD) under Basel accords. Also, the most accurate methodology for each exchange rate will be found and presented under the Mean Predictive Squared Error (MPSE).

\section{Conditional volatility modeling}

As we mentioned above, parametric univariate models are based on some features of in-sample distribution of portfolio returns. Three different specification are considered for each portfolio's time series conditional standard deviation $\sigma_{\mathrm{p}, \mathrm{t}}$ : the GARCH model (Bolleslev, 1986) the EGARCH model (Nelson, 1991) and the GJR-GARCH model (Glosten, Jaganathan, \&Runkle, 1993). In both models we consider their simplest form where the variance depends on one lag of past returns and past conditional variances.

\section{1 $\operatorname{GARCH}(1,1)$}

The GARCH(1,1) model is defined as: $\sigma_{p, t}^{2}=\omega+\alpha \varepsilon_{p, t-1}^{2}+\beta \sigma_{p, t-1}^{2}$ where $\omega>0, \alpha, \beta \geq 0$ and $\alpha+\beta<1$ to guarantee the positivity of conditional variances and stationarity of returns. The GARCH(p,q) model successfully captures several features of financial time series, such as thick tailed returns and volatility clustering.

\section{2 $\operatorname{EGARCH}(1,1)$}

Although GARCH(1,1) model captures the volatility clustering phenomenon and several features of financial time series, it could not explain the asymmetric relationship between returns and conditional variance. Nelson (1991) proposed another type of ARCH model, the exponential GARCH, or $\operatorname{EGARCH}(1,1)$ model that is defined as: $\operatorname{Ln}\left({\sigma_{p, t}^{2}}^{2}\right)=\omega+\beta \ln \left({\sigma_{p t-1}}^{2}\right)+\gamma \varepsilon_{t-1} /\left({\sigma_{p, t-1}}^{2}\right)^{1 / 2}+\alpha\left[\left|\varepsilon_{t-1}\right| /\left(\sigma_{p, t-1}\right)^{1 / 2}-(2 / \pi)^{1 / 2}\right]$ where the parameter $\gamma$ accommodates the asymmetric effect.

\subsection{GJR-GARCH(1,1)}

Glosten, Jaganathan $\kappa a l$ Runkle proposed another kind of GARCH-type model that it can explain the asymmetric relationship between returns and conditional variance and it can also capture the leverage effect, which may be a characteristic of several financial time series. GJR-GARCH modelis defined as: $\sigma_{t}^{2}=\alpha_{0}+\alpha_{1} \varepsilon^{2}{ }_{t-}$ ${ }_{1}+\beta \sigma_{t-1}^{2} \gamma \varepsilon_{t-1}^{2} I_{t-1}$ where $\mathrm{I}_{\mathrm{t}-1}=1$ if $\varepsilon_{\mathrm{t}-1}<0$ else $\mathrm{I}_{\mathrm{t}-1}=0$.

\section{Filtered Historical Simulation}

In the case of parametric methods, the distributional choice is crucial, while for non-parametric ones, we see there is no consistent method of estimating the volatility innovation. The Filtered Historical Simulation attempts to combine the advantages of the model-based with the advantages of the model-free approaches (HS) in a very intuitive fashion. So, FHS combines successfully the best elements of conditional volatility models with the best elements of the simulation methods. Using the quantiles of standardized residuals and the conditional standard deviation forecast from volatility models that are mentioned above, the VaR number is calculated as: $\operatorname{VaR}_{t+1}^{p}=-\sigma_{t+1}$ Percentile $\left\{\left\{z_{t+1-\tau}\right\}^{m}{ }_{t=1}, 100 p\right\}$. For empirical investigation purposes, we assume the volatility estimates and the corresponding quantiles are being generated via the $\operatorname{GARCH}(1,1)$, EGARCH(1,1) and GJR-GARCH(1,1) processes.

\section{Extreme Value Theory}

As we know, the biggest risk to a portfolio is a sudden occurrence of a single large negative return. Thus, risk managers should focus attention on modeling the tails of the returns distribution. The central result in extreme value theory states that the extreme tail of a wide range of distributions can be described by a relatively simple distribution, the so called generalized Pareto distribution. We apply the EVT method on standardized portfolio returns $\left(\varepsilon_{t}=y_{t} / \sigma_{t} \sim\right.$ iid.D $\left.(0,1)\right)$. Moreover, we filter the return series via the $\operatorname{GARCH}(1,1)$, EGARCH(1,1) and GJR-GARCH(1,1) processes in order to catch the empirical distribution. The probability

$$
\frac{\operatorname{Pr}\{u<z \leq x+u\}}{\operatorname{Pr}\{z>u\}}
$$

that standardized returns are greater than $\mathrm{u}$ is given by: $F_{u}(x)==\frac{F(x+u)-F(u)}{1-F(u)}$, where $\mathrm{x}>\mathrm{u}$. The

density function of GPD, $G(x ; \tau ; \beta)$ is described by: $G(x ; \tau ; \beta)=\left\{\begin{array}{c}1-\left(1+\frac{\tau x}{\beta}\right)^{-\frac{1}{\tau}}, \alpha v \tau \neq 0 \\ 1-\exp \left(-\frac{x}{\beta}\right)^{, \alpha v \tau=0} \text { and }\end{array}\right.$ 


$$
\begin{aligned}
& \left\{\begin{array}{c}
x \geq u, \quad \text { if } \tau \geq 0 \\
u \leq x \leq u-\frac{\beta}{\tau}, \quad \text { if } \tau<0
\end{array} \text {. Under the assumption of } \tau>0\right. \text {, a reasonable one for most financial time } \\
& \text { series, the Hill estimator }
\end{aligned}
$$

series, the Hill estimator

of the tail index $\tau$ equals: $\tau=1 / T_{u} \sum_{i=1}^{T_{u}} \ln \left(\frac{y_{i}}{u}\right)$

which is assumed to be equal to $5 \%$ of the total sample size $\mathrm{T}$. Thus, the $\mathrm{VaR}$ is calculated as: $\operatorname{VaR}_{t+1}^{p}=$ $\sigma_{P F, t+\mathbf{1}} F_{1-p}^{-\mathbf{1}}=\sigma_{P F, t+\mathbf{1}} u\left[\frac{p}{\frac{T_{u}}{T}}\right]^{-\xi}$, where $\sigma_{P F, t+\mathbf{1}}$ is the one-step-ahead conditional standard deviation forecast given by GARCH-type models.

\section{Evaluation framework}

Our objective is to test these different forecasting techniques, not in an econometric laboratory but in a risk management environment, so we must first choose a metric for the quality of such forecasts. It is known that there are a lot of sources of error in VaR figures, such as, data problems, faulty models, sampling errors etc. An adequate model must not only generate statistically accurate VaR values, but it also has to be 'preferred' over other equally adequate ones. There is a specific process for preferring the most appropriate $\mathrm{VaR}$ forecasting model.

\subsubsection{Backtesting VaR's}

Statistical adequacy will be tested based on Kupiec's and Christoffersen's backtesting measures, while the equivalent statistical adequacy of models will be performed via a statistical combined process. If we observe a time series of out-of-sample VaR forecasts and out-of-sample returns, we can define the 'hit sequence' of VaR violations as: $I_{t+1}=\left\{\begin{array}{ll}1, & \alpha v R_{P, t+1}<-V a R_{t+1}^{p} \\ 0, & \alpha v R_{P, t+1}>-V a R_{t+1}^{p} .\end{array}\right.$. The hit sequence of violations should be completely unpredictable and therefore distributed independently over time as a Bernoulli variable. To backtest VaR statistically, we use the three criteria by Christoffersen (1998). The first is the unconditional coverage test developed initially by Kupiec (1995). The null hypothesis is that the proportion of violations equals the expected one p. This is tested by a likelihood ration statistic. It's value $L_{\mathrm{uc}}$ is given by: $L R_{u c}=2 \ln \left[\left(1-\frac{N}{T}\right)^{T-N}\left(\llbracket \frac{N}{T}\right) \rrbracket^{N}\right]-2 \ln \left[(1-p)^{T-N} p^{N}\right] \sim X_{1}^{2}$

, where $\mathrm{N}$ is the number of days over the T out-of-sample days that a violation has occurred. The second criterion is the independence test. The null hypothesis is that the VaR failure process is independently distributed. This is also tested by a likelihood ration statistic. It's value $\mathrm{LR}_{\text {ind }}$ is given by: $L R_{\text {ind }}=2\left(\ln \left(\left(\llbracket 1-\pi_{01}\right) \rrbracket^{n_{00}} \pi_{01} n_{01}\left(\llbracket 1-\pi_{11}\right) \rrbracket^{n_{10} \pi_{11} n_{11}}\right)-\ln \left(\left(\llbracket 1-\pi_{0}\right) \rrbracket^{n_{00}+n_{10}} \pi_{0} n_{01}+n_{11}\right)\right) \sim X_{1}^{2}$

where $\mathrm{n}_{\mathrm{i}, \mathrm{j}}$ is the number of observations with value $\mathrm{i}$ followed by $\mathrm{j}$ gor $\mathrm{i}, \mathrm{j}=0,1$ and $\pi_{i, j}=\frac{n_{i, j}}{\sum_{j} n_{i, j}}$ are corresponding probabilities. The third criterion is the conditional coverage test that it's value $L_{\mathrm{cc}}$ is defined as: $L R_{c c}=L R_{u c}+L R_{i n d} \sim X_{2}^{2}$. The null hypothesis under this criterion is that the $\mathrm{VaR}$ violations are independent and the average number of violations is correct.

\subsubsection{Loss function}

In addition to backtesting measures outlined, a risk manager should also be able to evaluate the proposed models according to a utility function. An inherent problem of risk calculating models is that the 'true' VaR is never observed, not even after the realization of the actual return. Thus this kind of VaR can be proxied using the empirical distribution of realized returns. In our empirical analysis, 1000 observations are available for the outof-sample evaluation, their p-quantile will approximate the 'true' VaR. Thus, following the research of Diebold

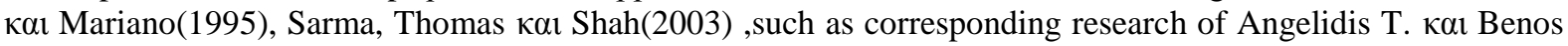
Alexandros, we use the loss function, named Quantile Loss $(\mathrm{QL})$, with the following form: 
, calculating the Mean Predictive Squared Error (MPSE), , in order to find the most efficient methodology for each exchange rate's VaR, which is used in the equation above.

$$
\begin{aligned}
& \Psi_{t+\mathbf{1}}^{Q L}= \begin{cases}\left(R_{P F, t+\mathbf{1}}-V a R_{t+\mathbf{1} \mid t}\right)^{2}, & \text { if } R_{P F, t+\mathbf{1}}<V a R_{t+\mathbf{1} \mid t} \\
\left(\text { Quantile }\left\{R_{P F}, 100 p\right\}_{1}^{T}-V a R_{t+\mathbf{1} \mid t}\right)^{2}, & \text { if } R_{P F, t+\mathbf{1}} \geq V a R_{t+\mathbf{1} \mid t}\end{cases} \\
& \Psi=T^{-\mathbf{1}} \sum_{t=1}^{T} \Psi_{t}^{Q L}
\end{aligned}
$$

5. Empirical Analysis

Our research focuses on logarithmic returns of the AUD/USD, EUR/JPY, EUR/USD and USD/CAD exchange rates extracted from yahoo finance. We have 4386 observations of logarithmic returns and we use a rolling sample of the last 1000 days $\log$ returns to forecast one day ahead $1 \%$ VaR estimates. Table 1 presents descriptive statistics for the analysis of the logarithmic returns of each exchange rate. We observe that the skewness is negative for AUD/USD, and EUR/JPY are positive for EUR/USD and USD/CAD. Likewise, kurtosis is higher than 3 for all time series.. We are thus confronted with a case of leptokurtic distributions, i.e. they have fat tails, in comparison with the normal distribution. These distributions do not follow normal distribution patterns confirmed by the Jarque-Bera test in Table 1. As we can say, the study of the behavior of the extremes in the left tails of these leptokurtic distributions seems justified as it should allow better estimation of extreme variations of financial returns. We chose a $95 \%$ quantile level as threshold, $u$ to fit the residuals from each GARCH-model to GPD. Under the framework of the loss function approach, we evaluate all the models with p-value greater than $10 \%$ for both unconditional and conditional coverage tests. Table 2 presents the results for Kupiec and Christoffersen tests. It seems that all of these exercised methods are located at the Green zone under Basel accords and most of them give efficient results on tests for significance level of $10 \%$. After calculating Mean Predictive Squared Error (MPSE) for all of these models for each exchange rate respectively, we present the results in Table 3.

\section{Conclusion}

The most frequently raised question in the finance literature is which model is to be used to predict the $\mathrm{VaR}$ of portfolio returns. Following the extensive and detailed investigation of some kinds of volatility modeling techniques and in combination with distribution approaches, briefly presented in the preceding sections, a number of comments are of order, aiming to summarize our results and give some fundamental guidelines with which to proceed in VaR estimation. Particular attention has been given to methodology based on Extreme Value Theory. The key argument of this paper is that after a combination of conditional volatility with FHS and EVT approaches, we evaluate these models after a procedure of backtesting, in which we find that most of the above models are correct under Basel accords. So, we implement a quantile loss function exercise that give us further information about the most accurate model in order to calculating one day VaR at $99 \%$ confidence level. Overall, the findings of this study imply that fat tails in return innovation process indeed play an important role in VaR estimates and should be considered in risk management. Thus, we should consider that GJR-EVT is the most accurate model for calculating VaR of AUD/USD, EUR/JPY, USD/CAD and FHSEGARCH is the most efficient for EUR/USD. In conclusion, we could say that GJR model with EVT approach for distribution captures most of the features of the above exchange rate time series and GJR-EVT model seem to be the most accurate model to forecasting VaR.

\section{Tables and Figures}

\begin{tabular}{|c|c|c|c|c|}
\hline & $D$ & $\bar{i}$ & $S$ & $s$ \\
\hline & $A U D / U S D$ & $E U R / J P Y$ & $E \quad U S R / C S S$ & $\begin{array}{lllllll}U & S & D & / & C & A & D\end{array}$ \\
\hline$M$ & $1,61 E-19$ & $3,59 E-19$ & $2,08 E-19$ & $-7,38000 E-20$ \\
\hline $\begin{array}{llllll}M & e & d & i & a & n\end{array}$ & 0,0000027 & 0,0000158 & $93 E-05$ & $8 \quad 8 \quad E \quad-\quad 0 \quad 5$ \\
\hline Max $\quad \boldsymbol{i} m u m$ & 0,056293 & 0,051703 & 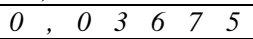 & $\begin{array}{llllll}0 & 3 & 2 & 7 & 5 & 5 \\
\end{array}$ \\
\hline$M i n i m u m$ & $-0,100652$ & $-0,073594$ & $-0,028194$ & $\begin{array}{cccccccc}-0 & 0 & 0 & 4 & 3 & 3 & 2 & 8 \\
\end{array}$ \\
\hline$S t d . \quad D e v$. & 0,0083008 & 0,007721 & $0,0,0 \begin{array}{llllll}0 & 0 & 6 & 3 & 1 & 3\end{array}$ & 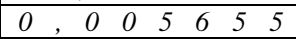 \\
\hline $\begin{array}{llllllll} & k & e & w & n & e & s & s\end{array}$ & $-0,805815$ & $-0,427521$ & $\begin{array}{llllll} & 3 & 4 & 2 & 3 & 8 \\
\end{array}$ & $\begin{array}{llllll}1 & 1 & 2 & 6 & 2 & 4 \\
\end{array}$ \\
\hline 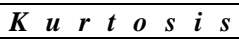 & 133,84214 & 9,29745 & $\begin{array}{llllll}6 & 7 & 7 & 2 & 2 & 5 \\
\end{array}$ & $\begin{array}{llllll}4 & 6 & 7 & 5 & 1 & 4 \\
\end{array}$ \\
\hline Jarque-Bera & 21957,28 & 7381,086 & $\begin{array}{llll}9 & 4 & 7 & 7\end{array}$ & $\begin{array}{llll}2 & 2 & 0 & 6 \\
\end{array}$ \\
\hline Probability & 0,0000000 & 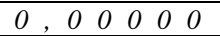 & $\begin{array}{lllll}0 & 0 & 0 & 0 & 0 \\
\end{array}$ & $\begin{array}{lllll}0 & 0 & 0 & 0 & 0 \\
\end{array}$ \\
\hline
\end{tabular}

Table 1: Descriptive statistics of four exchanges rates time series. 
Table 2: Results of Backtesting for Kupiec and Christoffersen tests for each model. The values in the parentheses correspond at the p-value which must compare to $1 \%, 5 \%, 10 \%$ respectively.

\begin{tabular}{|c|c|c|c|c|c|c|c|c|}
\hline & & $D$ & U & $D$ & & 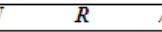 & & \\
\hline$M E \Gamma H O D S$ & $B A S E L$ & $\begin{array}{llll}L & R & u & c \\
\end{array}$ & $L R i n d$ & $\begin{array}{lll}L & R & c \\
\end{array}$ & $B A S E L$ & $\begin{array}{lll}L & R & u \\
\end{array}$ & $\begin{array}{lllll}L & R & i & n & d \\
\end{array}$ & $\begin{array}{lll}L & R & C \\
\end{array}$ \\
\hline \multirow[t]{2}{*}{ FHS-GARCH } & \multirow[t]{2}{*}{ GREEN } & $\begin{array}{llll}4 & 3 & 3 & 7 \\
\end{array}$ & 0,0000 & $\begin{array}{llll}4 & 3 & 3 & 7 \\
\end{array}$ & \multirow[t]{2}{*}{ GREEN } & $0,0,0000$ & $0,0,0000$ & $0,0,0000$ \\
\hline & & & $\left(1,0000^{888}\right)$ & $\left(0,8050^{888}\right)$ & & $\left(1,0000^{* 8 *}\right)$ & $\left(1,0000^{* 8 *}\right)$ & $\left(1,0000^{888}\right)$ \\
\hline \multirow[t]{2}{*}{ FHS-EGARCH } & \multirow[t]{2}{*}{$G R E E N$} & 70060 & 0,0000 & 70060 & \multirow[t]{2}{*}{$G R E E N$} & $0 \begin{array}{llll}0 & 1 & 5 & 6\end{array}$ & 00000 & $\begin{array}{llll}0 & 1 & 5 & 6\end{array}$ \\
\hline & & $\left(0,0300^{*}\right)$ & $\left(1,0000^{* 8 *}\right)$ & $(0,0950 * *)$ & & $\left(0,3140^{* 8 *}\right)$ & $\left(1,0000^{* 8 *}\right)$ & $\left(0,6020^{* * 8}\right)$ \\
\hline \multirow[t]{2}{*}{$F H S-G J R$} & \multirow[t]{2}{*}{$R E E N$} & 1,0156 & 0,0000 & 01156 & \multirow[t]{2}{*}{$G R E E N$} & 0156 & 00000 & 015 \\
\hline & & $\left(0,3140^{* 8 *}\right)$ & $\left(1,0000^{888}\right)$ & $\left(0,6020^{88 *}\right)$ & & $\left(0,3140^{* * *}\right)$ & $\left(1,0000^{* 88}\right)$ & $\left(0,6020^{* 88}\right)$ \\
\hline \multirow[t]{2}{*}{$G A R C H \cdot E V I$} & \multirow[t]{2}{*}{ GREEN } & $\begin{array}{llll}0 & 1 & 5 & 6 \\
\end{array}$ & 0,0000 & 1,01956 & \multirow[t]{2}{*}{ GREEN } & 00000 & 00000 & 00000 \\
\hline & & $\left(0,3140^{888}\right)$ & $\left(1,0000^{888}\right)$ & $\left(0,6020^{888}\right)$ & & $\left(1,0000^{8 * 8}\right)$ & $\left(1,0000^{888}\right)$ & $\left(1,0000^{888}\right)$ \\
\hline \multirow[t]{2}{*}{$E G A R C H \cdot E V T$} & \multirow[t]{2}{*}{$G R E E N$} & 3,00937 & 0,0000 & 3,00937 & \multirow[t]{2}{*}{ GREEN } & 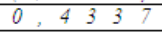 & $0 \begin{array}{llll}0 & 0 & 0 & 0 \\
\end{array}$ & $\begin{array}{llll}4 & 3 & 3 & 7 \\
\end{array}$ \\
\hline & & $(0,0790 * *)$ & $\left(1,0000^{8 * 8}\right)$ & $\left(0,2130^{* * *}\right)$ & & $\left(0,5100^{* 8 *}\right)$ & $\left(1,0000^{* * *}\right)$ & $\left(0,8050^{* 8 *}\right)$ \\
\hline \multirow[t]{3}{*}{$G J R-E V T$} & \multirow[t]{2}{*}{ GREEN } & $\begin{array}{llll}4 & 3 & 3 & 7\end{array}$ & 0,0000 & $\begin{array}{lll}4 & 3 & 3 \\
\end{array}$ & \multirow[t]{2}{*}{ GREEN } & 09978 & 00000 & 09 \\
\hline & & $\left(0,5100^{8 * 8}\right)$ & $\left(1,0000^{888}\right)$ & $\left(0,8050^{888}\right)$ & & $\left(0,7540^{* 8 *}\right)$ & $\left(1,0000^{88 *}\right)$ & $\left(0,9520^{* 8 *}\right)$ \\
\hline & & $R$ & $\bar{U}$ & $D$ & & $D$ & $C$ & $D$ \\
\hline MEГHODS & $E L$ & $R \quad u$ & $L R i n d$ & $L$ & $B A$ & $L$ & $R \quad i$ & $L$ \\
\hline \multirow[t]{2}{*}{ FHS-GARCH } & \multirow[t]{2}{*}{ GREEN } & $\begin{array}{llll}3 & 7 & 9 & 8 \\
\end{array}$ & 0,0000 & $\begin{array}{llll}3 & 7 & 9 & 8 \\
\end{array}$ & \multirow[t]{2}{*}{ GREEN } & $\begin{array}{llll}4 & 3 & 3 & 7 \\
\end{array}$ & $0,0,0000$ & $\begin{array}{llll}4 & 3 & 3 & 7 \\
\end{array}$ \\
\hline & & $\left(0,5380^{8 * 8}\right)$ & $\left(1,0000^{8 * 8}\right)$ & $\left(0,8270^{8 * *}\right)$ & & $\left(0,5100^{888}\right)$ & $\left(1,0000^{888}\right)$ & $\left(0,8050^{888}\right)$ \\
\hline \multirow[t]{2}{*}{ FHS-EGARCH } & \multirow[t]{2}{*}{$G R E E N$} & 0,09878 & 0,0000 & $\begin{array}{llll}0 & 9 & 7 & 8 \\
\end{array}$ & \multirow[t]{2}{*}{ GREEN } & 1,8862 & 0,00000 & 8862 \\
\hline & & $\left(0,7540^{888}\right)$ & $\left(1,0000^{* 88}\right)$ & $\left(0,9520^{888}\right)$ & & $\left(0,1700^{* * 8}\right)$ & $\left(1,0000^{888}\right)$ & $\left(0,3890^{* 88}\right)$ \\
\hline \multirow[t]{2}{*}{$F H S-G J R$} & \multirow[t]{2}{*}{ GREEN } & $0,0,9978$ & 0,0000 & $0,0,9878$ & \multirow[t]{2}{*}{ GREEN } & 1,00156 & 00000 & 01056 \\
\hline & & $\left(0,7640^{888}\right)$ & $\left(1,0000^{888}\right)$ & $\left(0,9520^{8 \times 8}\right)$ & & $\left(0,3140^{888}\right)$ & $\left(1,0000^{888}\right)$ & $\left(0,6020^{* 88}\right)$ \\
\hline \multirow[t]{2}{*}{ GARCH-EVT } & \multirow[t]{2}{*}{ GREEN } & 0,09978 & 0.0000 & 0.09978 & \multirow[t]{2}{*}{ GREEN } & 0,44337 & 0,00000 & 0,443337 \\
\hline & & $\left(0,7540^{* * 8}\right)$ & $\left(1,0000^{888}\right)$ & $(0,952088 *)$ & & $\left(0,5100^{8 * 8}\right)$ & $\left(1,0000^{* 8 *}\right)$ & $\left(0,8050^{8 * 8}\right)$ \\
\hline \multirow[t]{2}{*}{ EGARCH-EVT } & GREEN & $\begin{array}{llll}1 & 0 & 4 & 5 \\
\end{array}$ & 0,0000 & $\begin{array}{llll}1 & 0 & 4 & 5 \\
\end{array}$ & REEN & $\begin{array}{llll}0 & 9 & 3 & 7 \\
\end{array}$ & 00000 & $\begin{array}{llll}0 & 9 & 3 & 7 \\
\end{array}$ \\
\hline & & $\left(0,7460^{88 *}\right)$ & $\left(1,0000^{888}\right)$ & $\left(0,9490^{* 88}\right)$ & & $\left(0,0790^{* *}\right)$ & $\left(1,0000^{* 8 *}\right)$ & $\left(0,2130^{* * 8}\right)$ \\
\hline 1 & $E N$ & $\begin{array}{llll}0 & 9 & 7 & 8 \\
\end{array}$ & 0,0000 & $\begin{array}{llll}0 & 9 & 7 & 8 \\
\end{array}$ & $E N$ & 010156 & 00000 & 1 \\
\hline & & $\left(0,7540^{* 88}\right)$ & $\left(1,0000^{8 * 8}\right)$ & $(0,9520 * * 8)$ & & $\left(0,3140^{8 * 8}\right)$ & $\left(1,0000^{*}\right.$ & $\left(0,6020^{888}\right)$ \\
\hline
\end{tabular}

Table 3: Percentages of Mean Predictive Squared Error for exchange rates for each model respectively.

\begin{tabular}{|c|c|c|c|c|}
\hline $\boldsymbol{P}$ & $d$ & & & \\
\hline & $\begin{array}{lllllll}A & U & D & & U & S & D\end{array}$ & $E U R$ & $E U R / U S D$ & $U S D / C A D$ \\
\hline$F H S-G A R C H$ & $0,0.0011431 \% \%$ & $0,004091 \%$ & $0,001716 \%$ & 0 , \\
\hline$F H S-E G A R C H$ & $\begin{array}{lllllll}0 & 0 & 2 & 9 & 3 & 4 & \%\end{array}$ & $0,005112 \%$ & 0,00 & $\%$ \\
\hline$F \quad H$ & 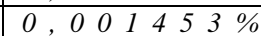 & $0,004303 \%$ & $0,001672 \%$ & $727 \%$ \\
\hline$E V T$ & $0,0014494 \%$ & $4084 \%$ & $0,001698 \%$ & $728 \%$ \\
\hline$E G A$ & 0,002 & $99 \%$ & 0,00 & 0,0 \\
\hline $\begin{array}{ll}G \quad J \\
\end{array}$ & 0 & 0,00 & $0,001646 \%$ & $0,000715 \%$ \\
\hline
\end{tabular}
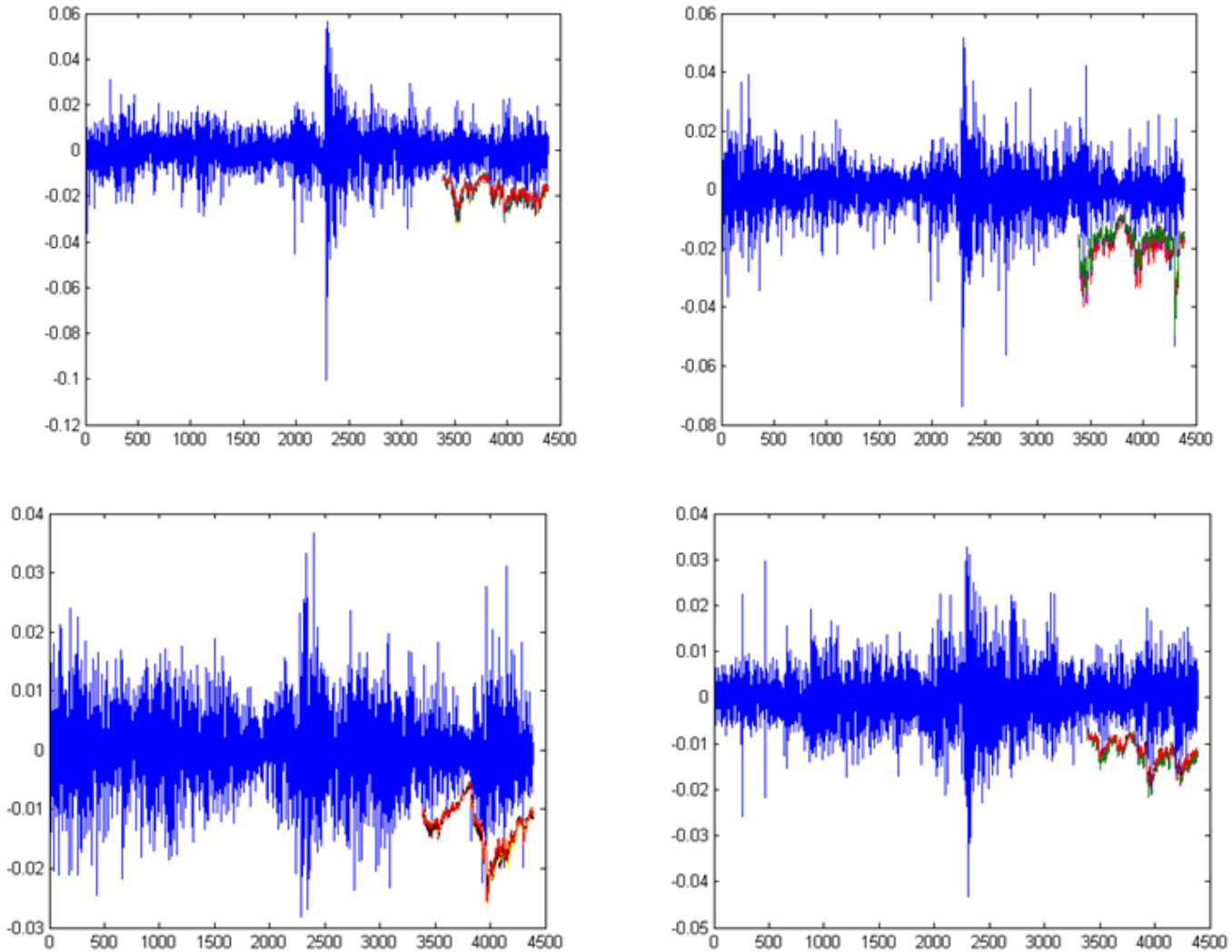

Figures: Returns Of Aud/Usd, Eur/Jpy, Eur/Usd, Usd/Cad And Out-Of-Sample Forecasts Of 99\% Var For Each Model. 


\section{References}

[1.] R. Alemany, C. Bolancé, M. Guillén, Nonparametric estimation of Value-at-Risk. Working Paper, Department of Econometrics, Riskcenter-IREA, University of Barcelona, 2012

[2.] Angelidis, T., Benos, A. and Degiannakis, S. 2004. The use of GARCH models in VaR estimation. Statistical Methodology Vol. 1, No. 2: 105-128.

[3.] Angelidis, T., Degiannakis, S. (2006), Backtesting VaR models: an expected shortfall approach, Working paper, Athens University of Economics and Business.

[4.] Assaf, A. (2009), Extreme observations and risk assessment in the equity markets of MENA region: Tail measures and Value-atRisk, International review of Financial Analysis, $\mathrm{N}^{\circ} 18,109-116$.

[5.] Barone-Adesi, G., Giannopoulos, K., Vosper, L. (2000), Filtered Historical Simulation.Backtest Analysis, University of Westminster.

[6.] Basel Committee on Banking Supervision, (2006), International Convergence of Capital Measurement and Capital standards: A Revised Framework Comprehensive Version (Bank for International Settlements, Basel).

[7.] Bollerslev, T. (1986), Generalised autoregressive conditional heteroskedasticity, Journal of Econometrics, 31, $309-328$.

[8.] Brooks, C., Persand, G., 2003. The effect of asymmetries on stock index return Value-at-Risk estimates. The Journal of Risk Finance, Winter, 29-42.

[9.] Christoffersen, P., Hahn, J., Inaoue, A. (2001), Testing and comparing value-at-risk measures, Journal of Empirical Finance, 8, 325-342.

[10.] Christoffersen, P. 2003. Elements of Financial Risk Management. Academic Press.

[11.] Coles, S. G. (2001). An introduction to Statistical Modelling of Extreme Values: Springer-Verlag.

[12.] Degiannakis, S., Xekalaki, E., 2004. Autoregressive Conditional Heteroscedasticity Models: A Review. Quality Technology and Quantitative Management, 1, 2, forthcoming.

[13.] Diebold, F.X., Mariano, R., 1995. Comparing predictive accuracy. Journal of Business and Economic Statistics, 13, 3, 253-263.

[14.] Dowd, K. (2005), Measuring market risk, Willey Finance.

[15.] Embrechts, P., Resnick, C., Samorodnitsky, G. (1999), Extreme Value Theory as a Risk Management Tool, North American Actuarial Journal, 3, 30-41.

[16.] Engle, Robert F., 1982, Autoregressive Conditional Heteroscedasticity with Estimates of the Variance of United Kingdom Inflation, Econometrica, 50(4), 987-1007.

[17.] Glosten, L., Jagannathan, R., Runkle, D., 1993. On the Relation Between the Expected Value and the Volatility of the Nominal Excess Return on Stocks. Journal of Finance, 48, 1779-1801.

[18.] J. Hull, A. White, Incorporating volatility updating into the historical simulation method for Value-at-Risk. Journal of Risk, Volume 1,1998

[19.] Jorion, P. (2002), Value at Risk, Second edition, McGraw Hill, New York.

[20.] Kupiec, P. (1995), Techniques for verifying the accuracy of risk management models, Journal of Derivatives, 3, 73-84.

[21.] Kourouma, L., Dupre, D., Sanfilippo, G., \& Taramasco, O. (2010). Extreme value at risk and expected shortfall during financial crisis.

[22.] McNeil A.J., Frey, R. (2000), Estimation of tail-related risk measures for heteroscedastic financial time series : an extreme value approach, Journal of Empirical Finance, 7, 271- 300.

[23.] Nelson, D., 1991. Conditional Heteroskedasticity in Asset Returns: A New Approach. Econometrica, 59, 347-370

[24.] Rozario, R. (2002). Estimating value at risk for the electricity market using a technique from extreme value theory, Working paper, University of New South Wales. 